This item was submitted to Loughborough's Research Repository by the author.

Items in Figshare are protected by copyright, with all rights reserved, unless otherwise indicated.

\title{
Fabrication of a polymeric optical waveguide-on-flex using electrostatic- induced lithography
}

PLEASE CITE THE PUBLISHED VERSION

http://dx.doi.org/10.1109/LPT.2010.2048310

PUBLISHER

(C) IEEE

VERSION

VoR (Version of Record)

LICENCE

CC BY-NC-ND 4.0

\section{REPOSITORY RECORD}

Hin, Tze Yang, Changqing Liu, Paul P. Conway, Weixing Yu, Scott Cargill, and Marc P.Y. Desmulliez. 2019. "Fabrication of a Polymeric Optical Waveguide-on-flex Using Electrostatic-induced Lithography". figshare. https://hdl.handle.net/2134/7137. 
This item was submitted to Loughborough's Institutional Repository (https://dspace.lboro.ac.uk/) by the author and is made available under the following Creative Commons Licence conditions.

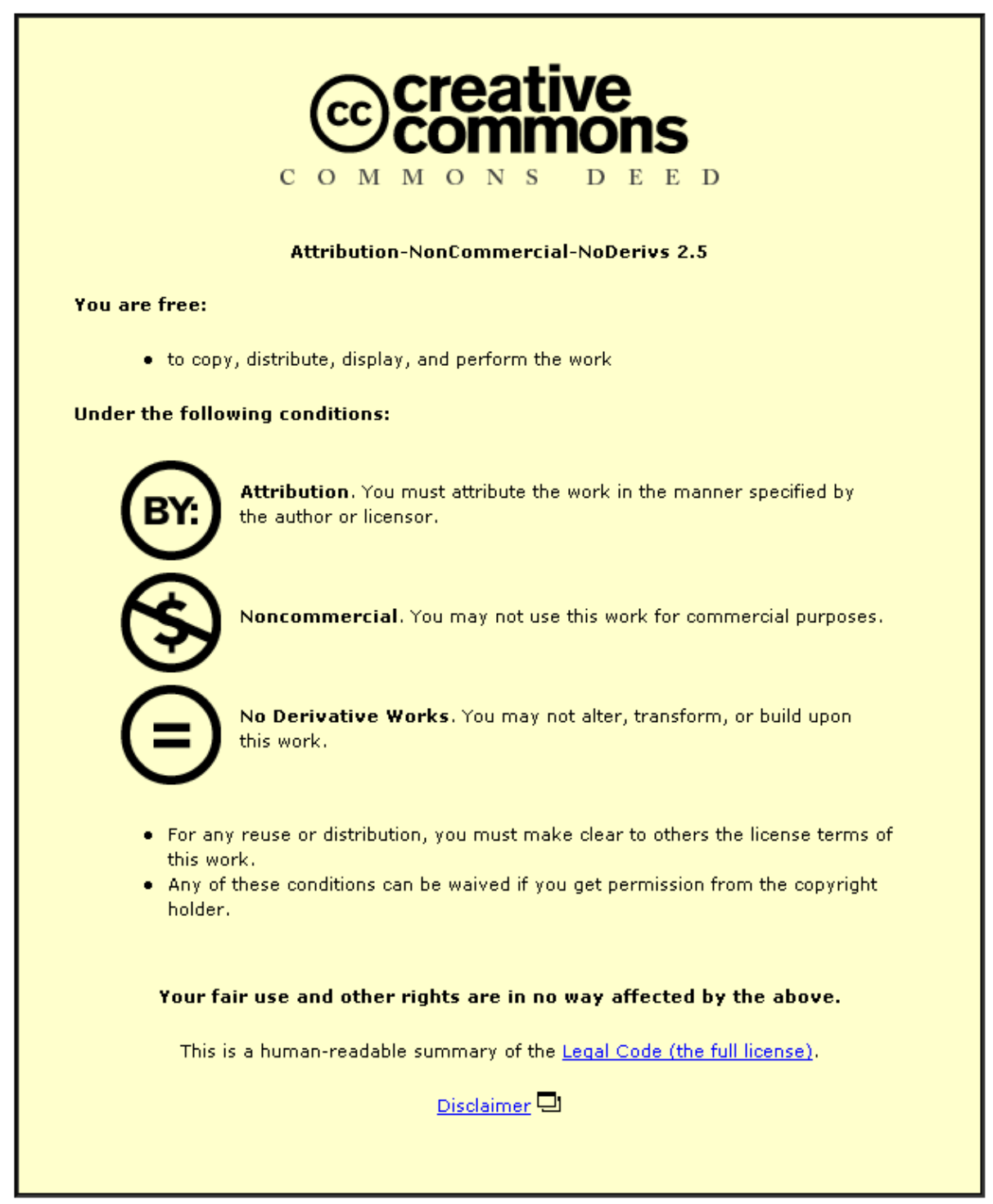

For the full text of this licence, please go to: http://creativecommons.org/licenses/by-nc-nd/2.5/ 


\title{
Fabrication of a Polymeric Optical Waveguide-On- Flex Using Electrostatic-Induced Lithography
}

\author{
Tze Yang Hin, Changqing Liu, Senior Member, IEEE, Paul P. Conway, Senior Member, IEEE, Weixing Yu, \\ Scott Cargill, and Marc P. Y. Desmulliez, Member, IEEE
}

\begin{abstract}
A method has been developed for the manufacture of polymeric multimode waveguides using an electrostatic field-induced self assembly and pattern formation process. A spin-coated liquid optical polymer placed between two conductive plates experiences an electrostatic force from an applied electric field gradient across the plates. Surface electrohydrodynamics instability patterning is employed to fabricate optical core microstructures using a patterned master plate. The result shows a good replication of the pattern from the master plate to the optical polymer. The process protocols were defined to achieve waveguides with low sidewall roughness together with an optical coupling interface. We have demonstrated multimode waveguide arrays with a $50 \mu \mathrm{m} \times 50 \mu \mathrm{m}$ cross section and $250-\mu \mathrm{m}$ pitch on a $10 \mathrm{~mm} \times 10 \mathrm{~mm}$ flexible substrate. The refractive index and absorption measurement of the electrostatic-induced optical film show insignificant changes when compared with the unexposed film. Using the cutback approach, the propagation loss of the waveguide is measured at $-1.97 \mathrm{~dB} / \mathrm{cm}$. The whole fabrication process is found to be fast, cost-effective, and no photosensitive material is needed as in the conventional photolithography approach.
\end{abstract}

Index Terms-Electrostatic-induced lithography, flexible substrate, optical core patterning, polymer waveguide.

\section{INTRODUCTION}

W ITH the introduction of multicore processor architecture, computing performance based on transistors and logic gates continues to grow according to Moore's law. An integrated electrical-optical interconnection technology is required that might incorporate the integration of optical channels, transceivers, connectors, and all interfacing to the multicore processor. This technology has been researched by leading semiconductor and optoelectronics companies and is called electrical-optical circuit board (EOCB) [1]. Recently, the development of optical waveguide-on-flex offers an attractive alternative to EOCB, allowing the folding and bending of light channels without the need for costly and inefficient light turning de-

Manuscript received January 20, 2010; revised March 08, 2010; accepted April 02, 2010. Date of publication April 19, 2010; date of current version June 09, 2010. This work was supported by the U.K. government's Engineering and Physical Sciences Research Council (EPSRC) funded Grand Challenge project "3D-Mintegration," referenced EP/C534212/1.

T. Y. Hin, C. Liu, and P. P. Conway are with Wolfson School of Mechanical and Manufacturing Engineering, Loughborough University, Loughborough LE11 3TU, U.K.

W. Yu is with Changchun Institute of Optics, Chinese Academy of Sciences, Changchun City, Jilin Province 130033, China.

S. Cargill and M. P. Y. Desmulliez are with Microsystems Engineering Center, Heriot-Watt University, Edinburgh EH14 4AS, U.K.

Color versions of one or more of the figures in this letter are available online at http://ieeexplore.ieee.org.

Digital Object Identifier 10.1109/LPT.2010.2048310

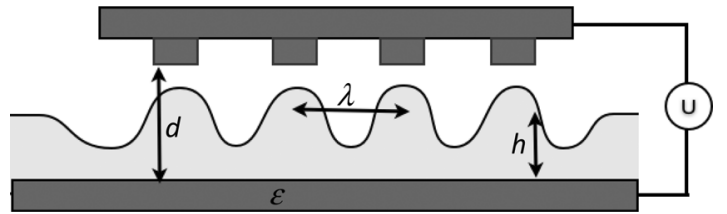

Fig. 1. Schematic illustration of a liquid polymer film destabilized by an electrostatic pressure induced by the applied electric field.

vices [1]. In this letter, we investigate the micro-patterning of liquid optical polymers on flexible circuit materials using electrostatic-induced lithography. Over the last decade, there has been much research on the formation of self-assembled or ordered structures in polymer thin films due to surface instabilities induced by electrostatic forces [2], [3]. Efforts have been undertaken to extend the electrohydrodynamics instability patterning (EHDIP) technique to the fabrication of microstructure patterns [4]-[6]. This work concentrates on the feasibility of this technology for the fabrication of low sidewall roughness optical core arrays in waveguide application at the microscale.

The concept of the EHDIP technique is illustrated schematically in Fig. 1. The polymer microstructures are formed by disturbing the dynamical surface stabilities of the spin-coated liquid core layer between two conductive plates. The growth of the liquid core structure is induced by an applied electric field, which overcomes the surface tension into a pattern predesigned on a metal electrode. Depending on the voltage $U$, the electrode spacing $d$, the initial film thickness $h_{0}$, the dielectric constant $\varepsilon$, and the surface tension $\gamma$, a characteristic wavelength of $\lambda$ is amplified. In a simplified description, the pattern formation of electrohydrodynamic instabilities is given in terms of a linear stability analysis [7].

For an applied voltage, $U$ in (1), the destabilizing pressure, $p$ in (2), scales with the square of the electric field strength in the polymer layer $E_{p}$

$$
E_{p}=\frac{U}{\varepsilon_{p} d-\left(\varepsilon_{p}-1\right) h}
$$

where

$$
p=-\varepsilon_{0} \varepsilon_{p}\left(\varepsilon_{p}-1\right) E_{p}^{2}
$$

and $\varepsilon_{0}$ is the dielectric permittivity in vacuum. For high enough fields, the electrostatic force overcomes gravity and other interfacial pressures [5], such as Van der Waals disjoining pressure or the Laplace pressure. Undulations start developing towards a liquid morphology spanning between the two electrodes, as shown in Fig. 2. Temporally, the amplitude of the fluctuations increases to a point where it induces a localized growth of a polymer structure. The presence of a pattern on one of the master 


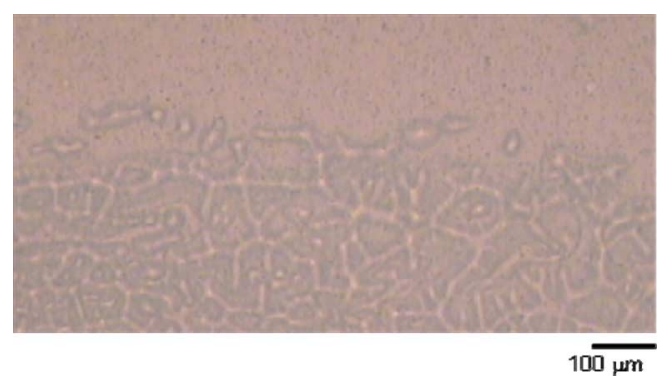

Fig. 2. Optical microscope image showing the overall morphology of a $15-\mu \mathrm{m}$-thick optical polymer coating. The lower part was exposed to an electric field $\left(U=0.9 \mathrm{kV}, d=60 \mu \mathrm{m}, h_{0}=15 \mu \mathrm{m}\right.$ ), showing typical initial modulations of the EHD instability.
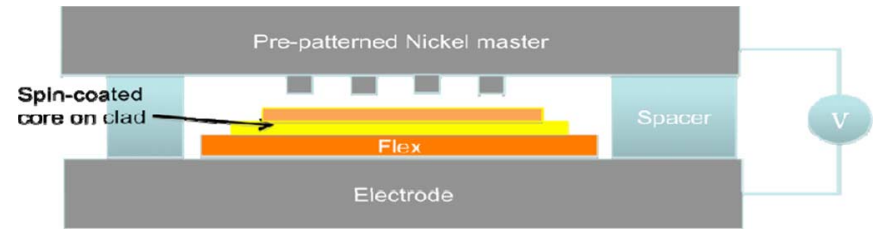

Fig. 3. Schematic of the experimental setup used for the fabrication of polymer waveguide arrays on flex.

electrode enables the creation of a nonuniform electrical field strength which enhances locally this instability pattern. From (1), the electric field strength of the polymer layer $E_{p}$ is increased with the increase of film thickness $h$.

\section{EXPERIMENT}

The optical core and cladding materials used in the waveguide production are a photothermal curing silsesquioxane LightLink (core $=$ XP-6701A and clad $=$ XP-07423A) produced by Rohm and Haas [8]. The processing and curing protocols of these materials are purposely altered to suit the EHDIP technique. Before applying the bottom cladding layers, the surface of the flexible substrate KaptonHN is plasma-treated. The optical layers are applied by spin-coating. Upon curing of the cladding layer, the optical core is spin-coated to obtain a thick coating range at $10-15 \mu \mathrm{m}$. A soft bake step at $40{ }^{\circ} \mathrm{C}$ is used to remove the majority of the solvent (i.e., PGMEA) before the EHDIP process commences.

Fig. 3 illustrates the experimental setup of the EHDIP process. A nickel-based plate with waveguide arrays pattern fabricated by an electroforming process is used as a master. The grounded bottom electrode is a simple flat sheet metal to provide rigid support for the flexible substrate. The initial gap between the master and the top surface of optical polymer is determined by the spacer to provide a path for surface evolution. It has been predicted that the applied electric fields required across this capacitor like arrangement to induce polymeric microstructure growth are at the order of $10^{7} \mathrm{Vm}^{-1}$ [3]. In this experiment, the electrical range of $0.5-1.5 \mathrm{kV}$ is applied at a gap of 30-60 $\mu \mathrm{m}$. The liquid polymer becomes attracted by the extruded pattern on the master electrode and tends over time to replicate its patterns. The sample is left in these conditions for 15-60 min before the applied field is removed and subsequently exposed to UV and heat curing. The sample is cured with UV radiation of $5 \mathrm{~mW} / \mathrm{cm}^{2}$ for $100 \mathrm{~s}$ and hard baked at $145^{\circ} \mathrm{C}$ for $15 \mathrm{~min}$ on a hot plate. Finally, the sample is coated by the top cladding to complete the waveguide-on-flex fabrication.

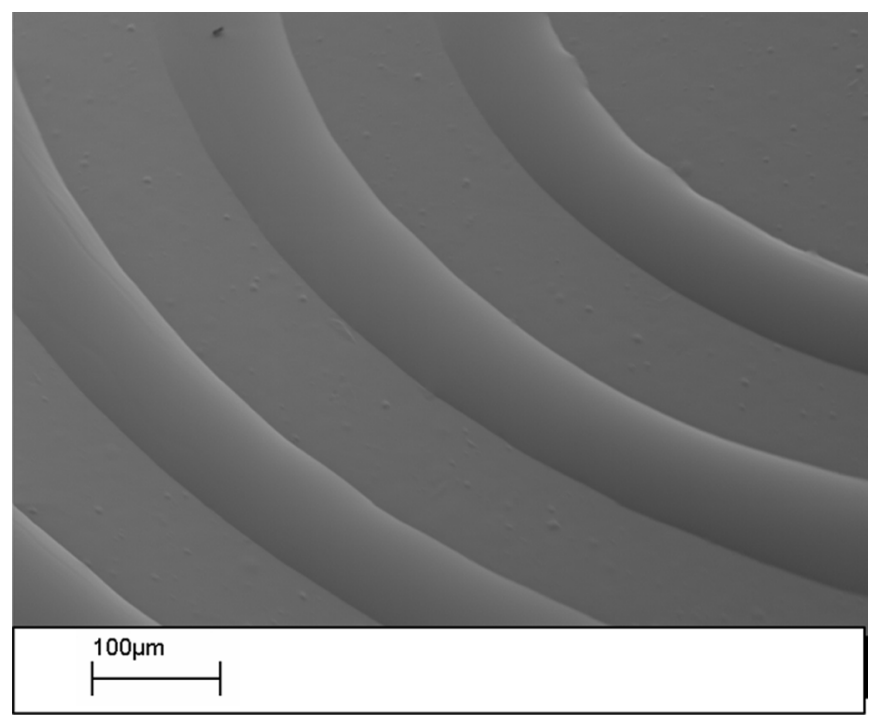

Fig. 4. SEM of the fabricated waveguide structures with de voltage of $0.9 \mathrm{kV}$. Prebake excluded, $h_{0}=10 \mu \mathrm{m}, d=50 \mu \mathrm{m}$.

\section{RESUlT AND DISCUSSION}

Three different cases of process parameters were studied in the electroswelling of the liquid photopolymer, allowing the shape control of the microstructure formation. In the first case, the spin-coated optical core film was subjected directly to an applied voltage of $0.9 \mathrm{kV}$. Fig. 4 shows the resulting weakly formed structures. Upon the removal of electrostatic field, the low viscosity nature of polymeric structure leads to the spreading of the liquid on the deposited surface. At the current process capability, the electrostatic-induced microstructures are not cured with the field intact. Consequently, the flat tops are not obtained due to the spreading of the liquid in the course of curing.

In order to partially remove unnecessary solvent of the liquid, a soft bake step is included in the second case before electroswelling. While maintaining the similar initial spin-coated film thickness $h_{0}$ of $10 \mu \mathrm{m}$, Fig. 5(a) and (b) shows the semispherical waveguide structures obtained due to the limited spread as a result of the more viscous polymer solution. Such semispherical structures may be advantageous for optical interface coupling with circular fiber facet. The discontinuity structures formed is believed to be caused by the low initial spin-coated thickness and the large variation in the manually applied electrostatic field.

Third, an array of steep sidewall waveguide structures is successfully achieved as shown in Fig. 5(c) and (d) with the inclusion of a soft bake step and the increase of the initial film thickness, $h_{0}$ of $15 \mu \mathrm{m}$. Indeed, the polymer can be seen to have touched the top electrodes where the top surface is flat. The undulation of the sidewall occurred during the separation of master surface from the polymer. Such undulation increases the sidewall surface roughness and contributes to optical scattering loss. However, we believe that the release of the waveguide structures from the master can be improved by employing surface release agent on the master surface. Nonetheless, the EHDIP process is able to produce a very smooth surface finish, as shown in Fig. 5(b) and (d).

The effect of electrostatic field exposure to the optical properties of polymer films was investigated. The refractive indices 


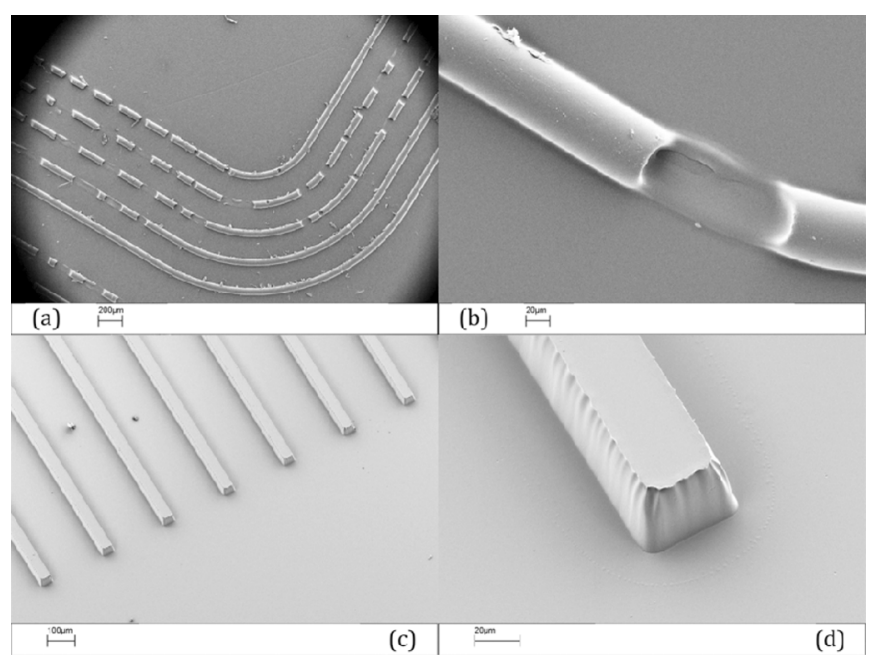

Fig. 5. SEM of the fabricated waveguide structures with dc voltage of $0.9 \mathrm{kV}$. Prebake included, $d=50 \mu \mathrm{m}$. (a), (b) $h_{0}$ at $10 \mu \mathrm{m}$; (c), (d) $h_{0}$ at $15 \mu \mathrm{m}$.

TABLE I

REFRACTIVE INDEX MEASUREMENT OF LIGHTLINK CORE MATERIAL IS COMPARED TO THE EleCtrostatic Field EXPOSED SAMPLE

\begin{tabular}{rcc} 
& On KaptonHN $\mathrm{N}^{\mathrm{TM}}$ & Free standing film \\
\hline Normal procedure: Unexposed & 1.5152 & 1.5161 \\
& 1.5156 & 1.5160 \\
Average & 1.5154 & 1.5161 \\
\hline Exposed to electrostatic field & 1.5150 & 1.5162 \\
& 1.5150 & 1.5163 \\
Average & 1.5150 & 1.5163 \\
\hline
\end{tabular}

of both optical films (cured with normal procedure and exposed to electrostatic field) deposited on flex substrate were measured using Metricon unit at $847 \mathrm{~nm}$. The refractive indices of the samples were measured first on the substrate, before the coatings were removed and measured separately for comparison. The result in Table I shows a $0.5 \%$ higher refractive index on the free standing film when compared to measurement on the substrate. However, the slight change with the exposed and unexposed samples observed at less than $0.03 \%$ is of no critical interest to the waveguide design, which has $2 \%$ difference between core and clad layers.

Lightlink polymer is known to be highly transparent in the visible and near-infrared region. Fig. 6 shows the identical absorbance spectra of the processed samples with and without electrostatic field exposure. One sample was exposed to an electric field of $0.9 \mathrm{kV}$ at a $50-\mu \mathrm{m}$ gap for $15 \mathrm{~min}$ before cured with the normal procedure. It is concluded that the electroswelling process introduced no unwanted intrinsic absorption.

The propagation loss of the fabricated waveguide was measured using the cutback approach. A laser source was coupled into the waveguide using 50/125 $\mu$ m multimode fiber assisted by a three-directional charged-coupled device (CCD) visual alignment system. The output power at wavelength $850 \mathrm{~nm}$ was used. The transmitted light through the waveguide was coupled into a photodetector via an intermediate optical fiber.

The coupling losses of the waveguide was measured at $2,1.5$, and $1.0 \mathrm{~cm}$ accordingly. The propagation loss of the waveguide system calculated based on the cutback approach

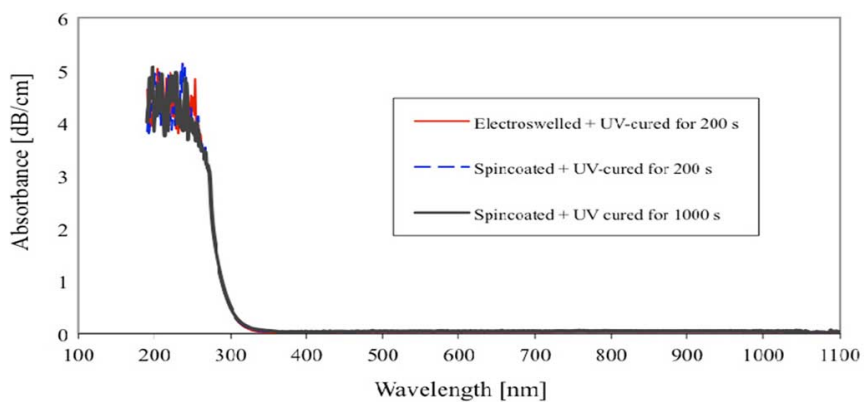

Fig. 6. Comparison of UV-visible absorbance spectra of Lightlink core with and without exposure to electrostatic field measured using UV-visible double beam spectrometer.

is $-1.97 \mathrm{~dB} / \mathrm{cm}$. The excessive attenuation indicates that the manually fabricated waveguide is not ready for the industrial application. Further process optimization is required to reduce defects such as contaminants, voids, microcracks, and material shrinkage.

\section{CONCLUSION}

We are reporting an array of optical polymeric waveguide microstructures fabricated using electrostatic field induced lithography. Using the EHDIP technique, various waveguide core profiles were obtained by altering the solvent/solid ratio of the optical polymer and initial spin-coated liquid photopolymer thickness. In addition, initial optical characterization reveals that no unwanted change to the refractive index and absorption of the optical core material upon exposure to the electrostatic field. The propagation loss of the fabricated waveguide is calculated at $1.97 \mathrm{~dB} / \mathrm{cm}$.

\section{ACKNOWLEDGMENT}

The refractive index measurement is the courtesy of Dow Chemical.

\section{REFERENCES}

[1] R. Dangel, R. Beyeler, N. Meier, T. Lamprecht, F. Horst, D. Jubin, J. Weiss, and B. J. Offrein, "Optical interconnects for board level applications," Optoelectronic Integrated Circuits XI. San Jose, CA, 2009, pp. 721904-721907.

[2] S. Herminghaus, "Dynamical instability of thin liquid films between conducting media," Phys. Rev. Lett., vol. 83, p. 2359, 1999.

[3] E. Schaffer, T. Thurn-Albrecht, T. P. Russell, and U. Steiner, "Electrically induced structure formation and pattern transfer," Nature, vol. 403, pp. 874-877, 2000.

[4] M. Yang, C. S. Ozkan, and H. Gao, "Self assembly of polymer structures induced by electric field," J. Assoc. Laboratory Automation, vol. 8, pp. 86-89, 2003.

[5] N. Wu and W. B. Russel, "Dynamics of the formation of polymeric microstructures induced by electrohydrodynamic instability," Appl. Phys. Lett., vol. 86, pp. 241912-241913, 2005.

[6] W. Yu, S. Cargill, M. Leonard, and M. P. Y. Desmulliez, "Micro-fabrication on 3-D surface by electrostatic induced lithography," in Proc. 2nd Electronics Systemintegration Technology Conf., 2008 (ESTC 2008), pp. 111-116.

[7] L. F. Pease and W. B. Russel, "Limitations on length scales for electrostatically induced submicrometer pillars and holes," Langmuir, vol. 20, pp. 795-804, 2004.

[8] E. Anzures, R. Dangel, R. Beyeler, A. Cannon, F. Horst, C. Kiarie, P. Knudsen, N. Meier, M. Moynihan, and B. J. Offrein, "Flexible optical interconnects based on silicon-containing polymers," presented at the Photonics Packaging, Integration, and Interconnects IX, San Jose, CA, 2009, 72210I-12. 